\title{
Monolithic, Ultra-Thin GalnP/GaAs/GalnAs Tandem Solar Cells
}

Mark Wanlass, Phil Ahrenkiel, David Albin, Jeff Carapella, Anna Duda, Keith Emery, Daniel Friedman, John Geisz, Kim Jones, Alan Kibbler, James Kiehl, Sarah Kurtz, William McMahon, Tom Moriarty, Jerry Olson, Aaron Ptak, Manuel Romero, and Scott Ward

National Renewable Energy Laboratory (NREL), 1617 Cole BIvd., Golden, CO, USA

Email: mark wanlass@nrel.gov; Phone: (303) 384-6532; Fax: (303) 384-6430

Presented at the 2006 IEEE 4th World Conference on Photovoltaic Energy Conversion (WCPEC-4) held May 7-12, 2006 in Waikoloa, Hawaii. 


\section{Disclaimer and Government License}

This work has been authored by Midwest Research Institute (MRI) under Contract No. DE-AC36-99GO10337 with the U.S.

Department of Energy (the "DOE"). The United States Government (the "Government") retains and the publisher, by accepting the work for publication, acknowledges that the Government retains a non-exclusive, paid-up, irrevocable, worldwide license to publish or reproduce the published form of this work, or allow others to do so, for Government purposes.

Neither MRI, the DOE, the Government, nor any other agency thereof, nor any of their employees, makes any warranty, express or implied, or assumes any liability or responsibility for the accuracy, completeness, or usefulness of any information, apparatus, product, or process disclosed, or represents that its use would not infringe any privately owned rights. Reference herein to any specific commercial product, process, or service by trade name, trademark, manufacturer, or otherwise does not constitute or imply its endorsement, recommendation, or favoring by the Government or any agency thereof. The views and opinions of the authors and/or presenters expressed herein do not necessarily state or reflect those of MRI, the DOE, the Government, or any agency thereof. 


\section{Monolithic, Ultra-Thin GalnP/GaAs/GalnAs Tandem Solar Cells}

- NREL IR \# 05-05, patent pending.

- Near-optimum subcell bandgaps.

- $300 \mathrm{mV}$ voltage output boost compared to conventional Ge-based triple-junction tandems.

- Bottom subcell $\mathrm{E}_{\mathrm{g}}$ is variable.

- Basic approach is expandable to 4-6 subcells.

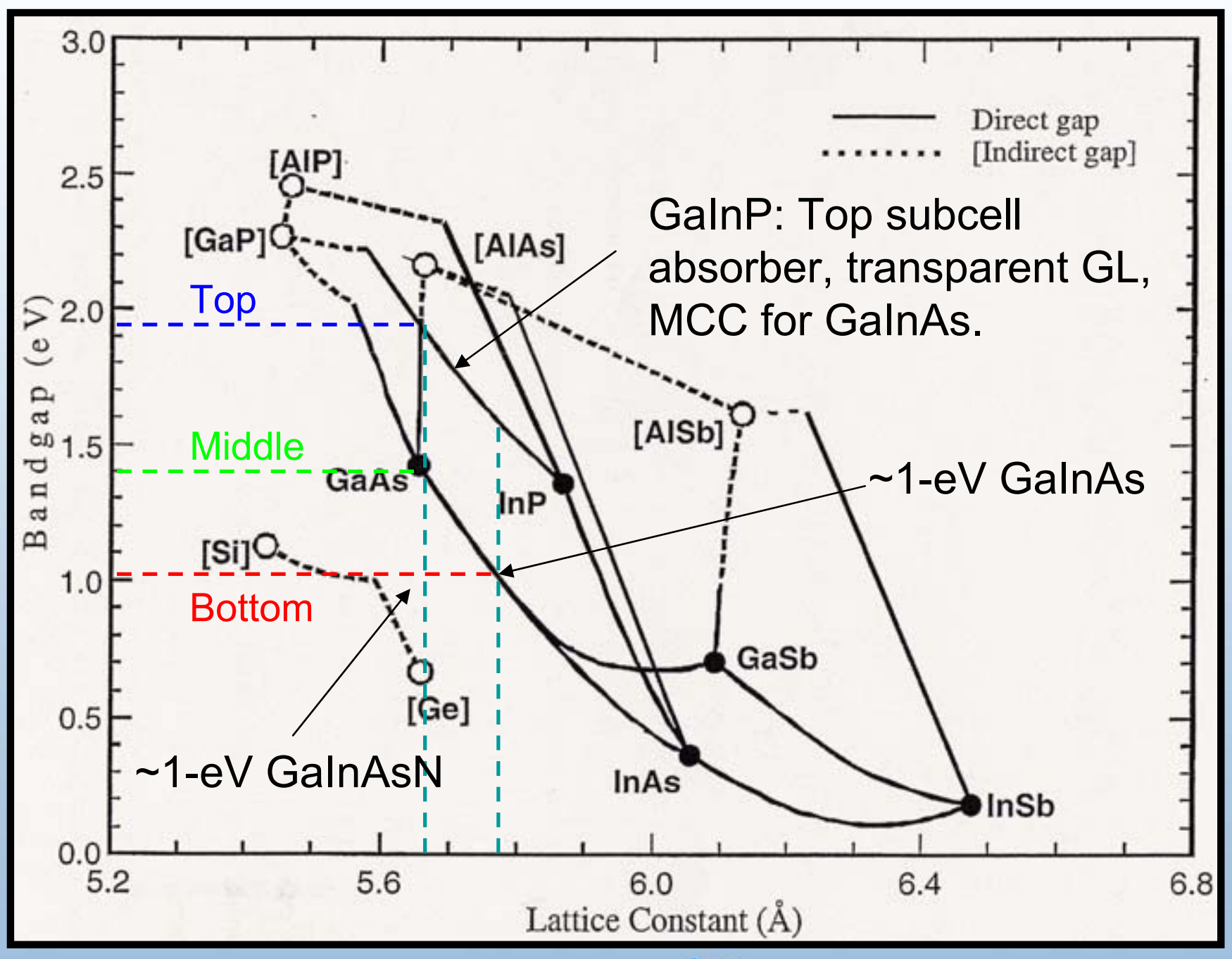

NREL is operated by Midwest Research Institute. Battelle

MWW 2006 WCPEC-4 


\section{Inverted GalnP/GaAs/GalnAs Tandem Structure}

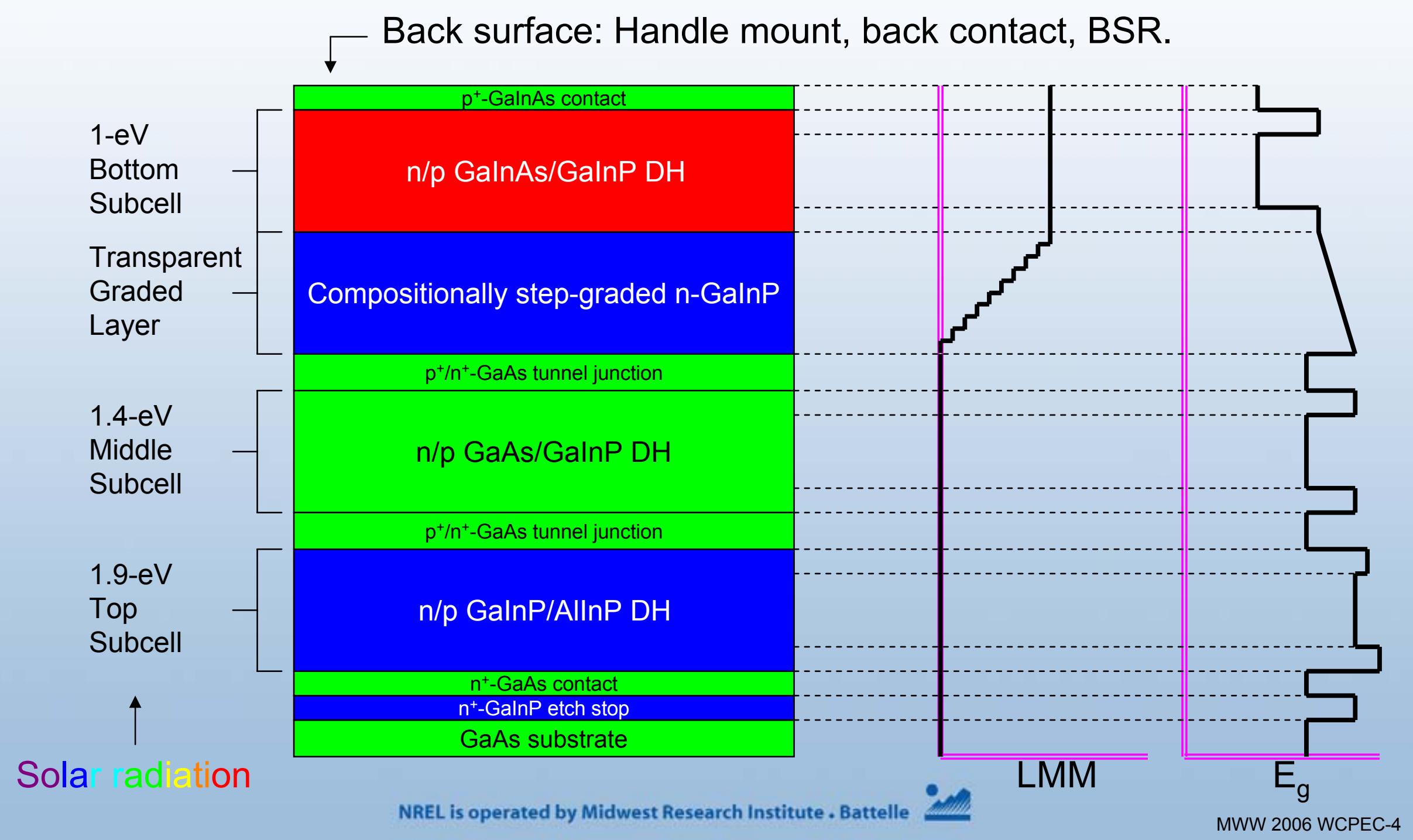




\section{Ultra-thin Tandem Cell Processing Sequence}

\begin{tabular}{|c|}
\hline Back contact/BSR \\
\hline Contact layer \\
\hline Multi-bandgap device layers \\
\hline Contact layer \\
\hline Etch-stop layer \\
\hline Parent substrate \\
\hline
\end{tabular}

1) Inverted tandem structure is grown on the parent substrate, and the back contact/BSR is formed.

\begin{tabular}{|c|}
\hline Contact layer \\
\hline Multi-bandgap device layers \\
\hline Contact layer \\
\hline Back contact/BSR \\
\hline Handle (support substrate)
\end{tabular}

3) Parent substrate is removed.
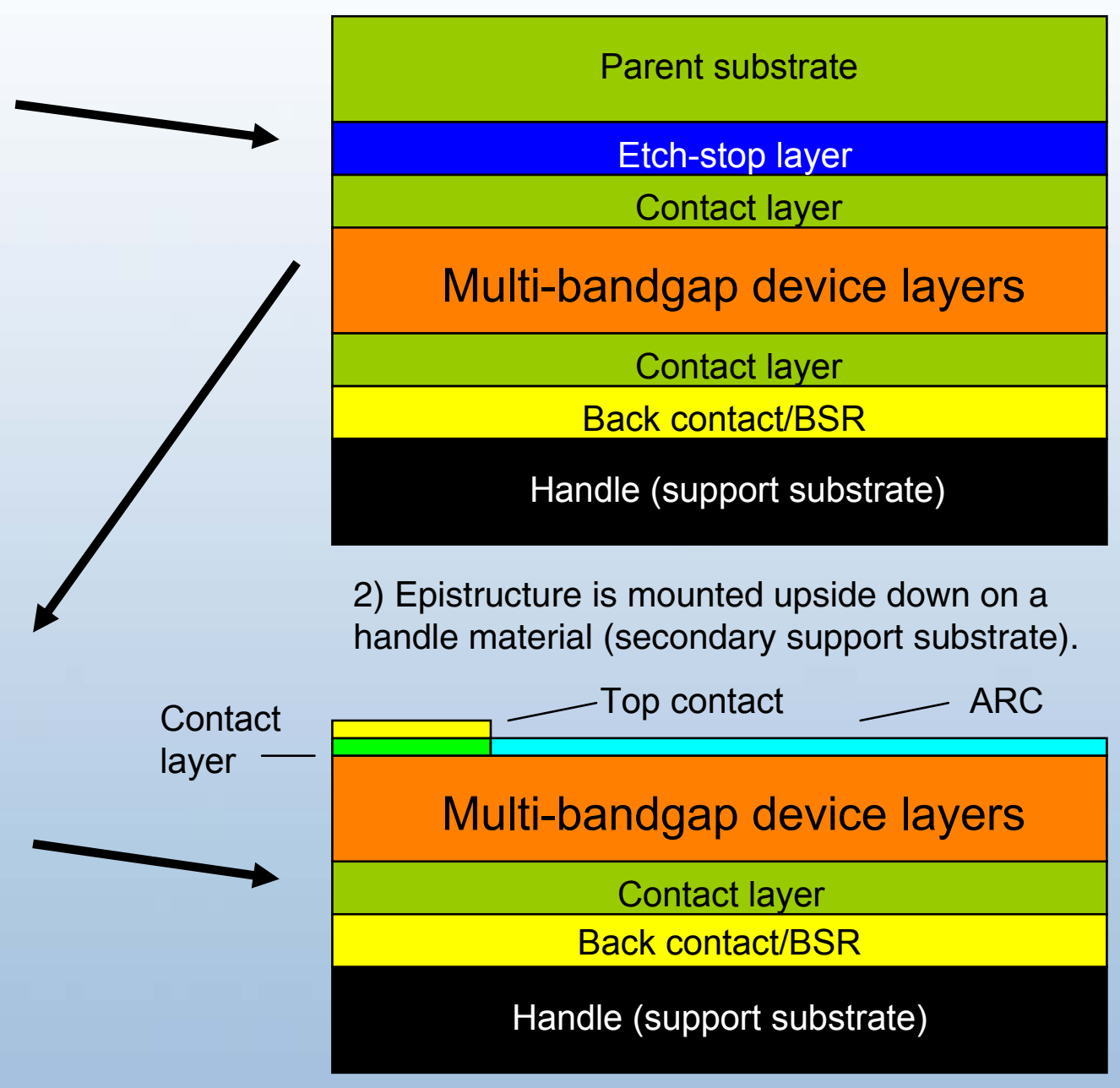

4) Front-surface processing is completed.

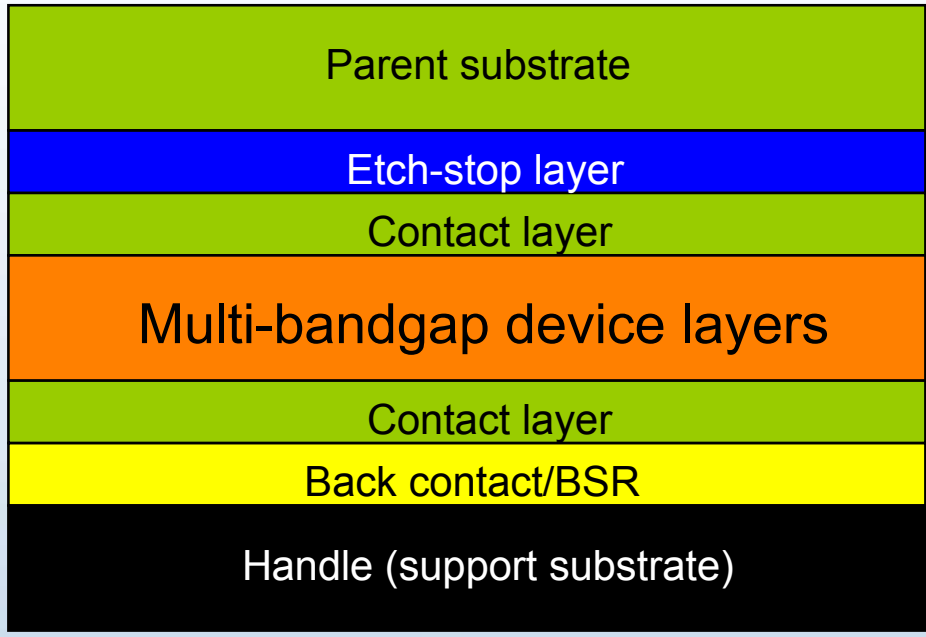

2) Epistructure is mounted upside down on a handle material (secondary support substrate). 


\section{NPE 1 National Renewable Energy Laboratory}

\section{Advantages of Ultra-Thin, Handle- Mounted Tandem Solar Cells}

- Handle material can be engineered to have a wide range of advantageous characteristics.

- Thermal management can be optimized.

- Highest specific power (W/kg) for space applications.

- Reuse and/or reclamation of the parent substrate also possible, reducing cost.

- Parent substrate can be impure to reduce cost.

- Benefits of BSR: thin GalnAs subcell, lower $J_{0}$, improved radiation hardness, reduced operating temperature. 


\section{Semi-Realistic Performance Modeling GalnP/GaAs/GalnAs}

Low-AOD Direct Spectrum, 250 suns, $25^{\circ} \mathrm{C}$ $\mathrm{QE}=0.95$, realistic $J_{0}\left(E_{\mathrm{g}}\right)$, no parasitic losses

Subcell parameters

\begin{tabular}{|l|l|l|l|l|}
\hline $\begin{array}{l}\text { Subcell } \\
\text { Absorber }\end{array}$ & $\mathrm{E}_{\mathrm{g}}(\mathrm{eV})$ & $\mathrm{V}_{\mathrm{oc}}(\mathrm{V})$ & $\begin{array}{l}\mathrm{J}_{\mathrm{sc}} \\
\left(\mathrm{A} / \mathrm{cm}^{2}\right)\end{array}$ & $\mathrm{FF}(\%)$ \\
\hline GalnP & 1.87 & 1.53 & 3.40 & 91.57 \\
\hline GaAs & 1.42 & 1.12 & 3.40 & 89.23 \\
\hline GalnAs & 1.01 & 0.74 & 3.63 & 85.21 \\
\hline
\end{tabular}

Series-connected tandem parameters

\begin{tabular}{|l|l|l|l|l|l|}
\hline $\mathrm{V}_{\text {oc }}(\mathrm{V})$ & $\begin{array}{l}\mathrm{Jsc} \\
\left(\mathrm{A} / \mathrm{cm}^{2}\right)\end{array}$ & $\begin{array}{l}\mathrm{FF} \\
(\%)\end{array}$ & $\begin{array}{l}\mathrm{V}_{\max } \\
(\mathrm{V})\end{array}$ & $\begin{array}{l}\mathrm{J}_{\max } \\
\left(\mathrm{A} / \mathrm{cm}^{2}\right)\end{array}$ & $\begin{array}{l}\mathrm{P}_{\max } \\
\left(\mathrm{W} / \mathrm{cm}^{2}\right)\end{array}$ \\
\hline 3.38 & 3.40 & 90.32 & 3.11 & 3.34 & 10.38 \\
\hline
\end{tabular}

Tandem efficiency: $41.5 \%$ 


\section{Semi-Realistic Performance Modeling}

Subcell parameters

Conditions

AM0, one sun, $25^{\circ} \mathrm{C}$

$\mathrm{QE}=0.95$, Realistic $\mathrm{J}_{0}\left(\mathrm{E}_{\mathrm{g}}\right)$

\begin{tabular}{|l|l|l|l|l|}
\hline $\begin{array}{l}\text { Subcell } \\
\text { Absorber }\end{array}$ & $\mathrm{E}_{\mathrm{g}}(\mathrm{eV})$ & $\mathrm{V}_{\mathrm{oc}}(\mathrm{V})$ & $\begin{array}{l}\mathrm{J}_{\text {sc }} \\
\left(\mathrm{mA} / \mathrm{cm}^{2}\right)\end{array}$ & $\mathrm{FF}(\%)$ \\
\hline GalnP & 1.87 & 1.393 & 17.00 & 90.94 \\
\hline GaAs & 1.42 & 0.981 & 17.00 & 88.09 \\
\hline GalnAs & 1.02 & 0.608 & 18.13 & 83.01 \\
\hline
\end{tabular}

Tandem efficiency

$\sim 33 \%$ (one sun)

$\sim 36 \%$ (10 suns)

Series-connected tandem parameters

\begin{tabular}{|l|l|l|l|l|l|}
\hline $\mathrm{V}_{\text {oc }}(\mathrm{V})$ & $\begin{array}{l}\mathrm{Jsc} \\
\left(\mathrm{mA} / \mathrm{cm}^{2}\right)\end{array}$ & $\begin{array}{l}\mathrm{FF} \\
(\%)\end{array}$ & $\begin{array}{l}\mathrm{V}_{\max } \\
(\mathrm{V})\end{array}$ & $\begin{array}{l}\mathrm{J}_{\max } \\
\left(\mathrm{mA} / \mathrm{cm}^{2}\right)\end{array}$ & $\begin{array}{l}\mathrm{P}_{\max } \\
\left(\mathrm{mW} / \mathrm{cm}^{2}\right)\end{array}$ \\
\hline 2.98 & 17.00 & 89.27 & 2.72 & 16.65 & 45.26 \\
\hline
\end{tabular}




\section{Defects in LMM, 1-eV GalnAs}

\section{XTEM}

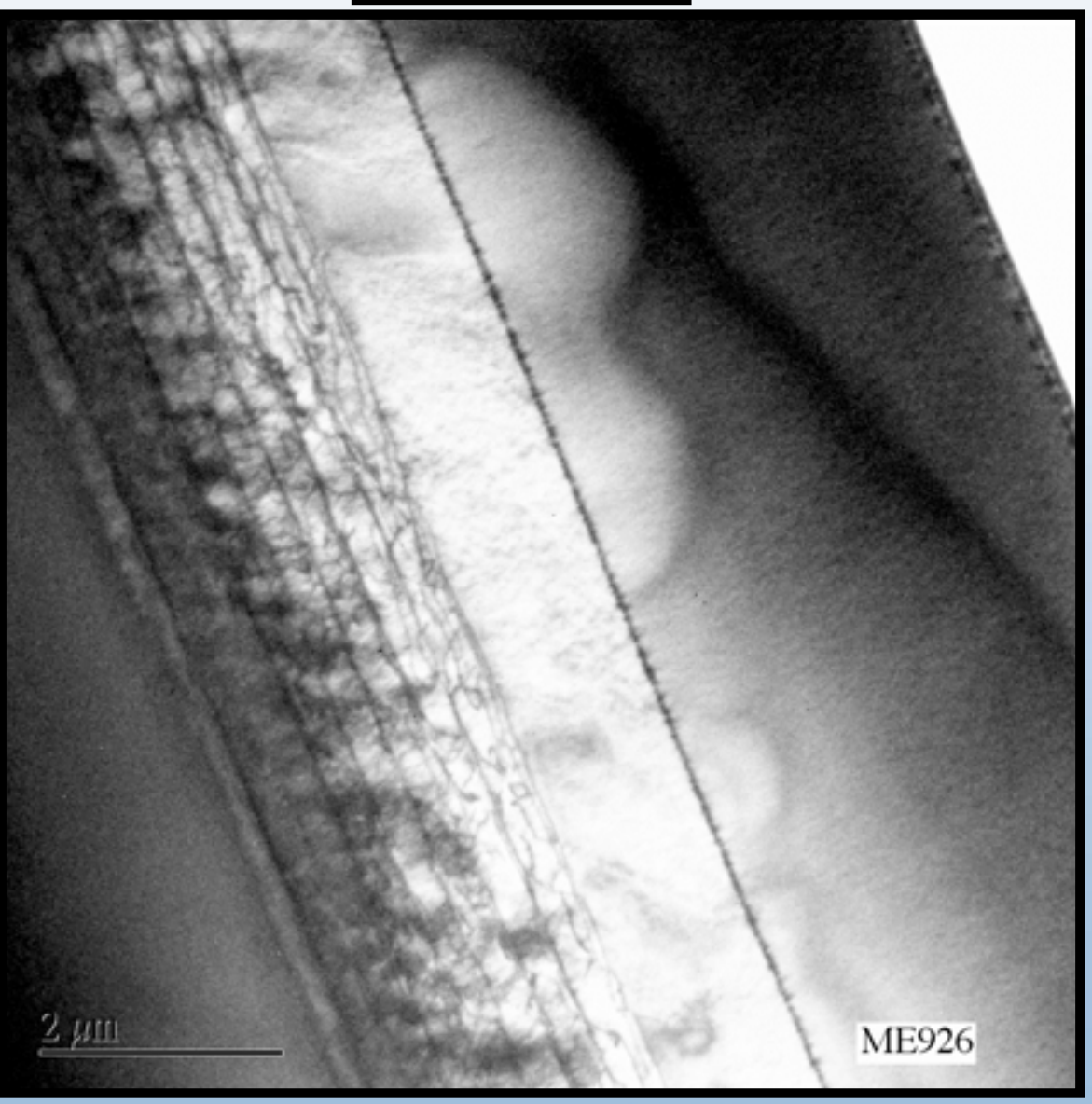

\section{Plan-view CL}

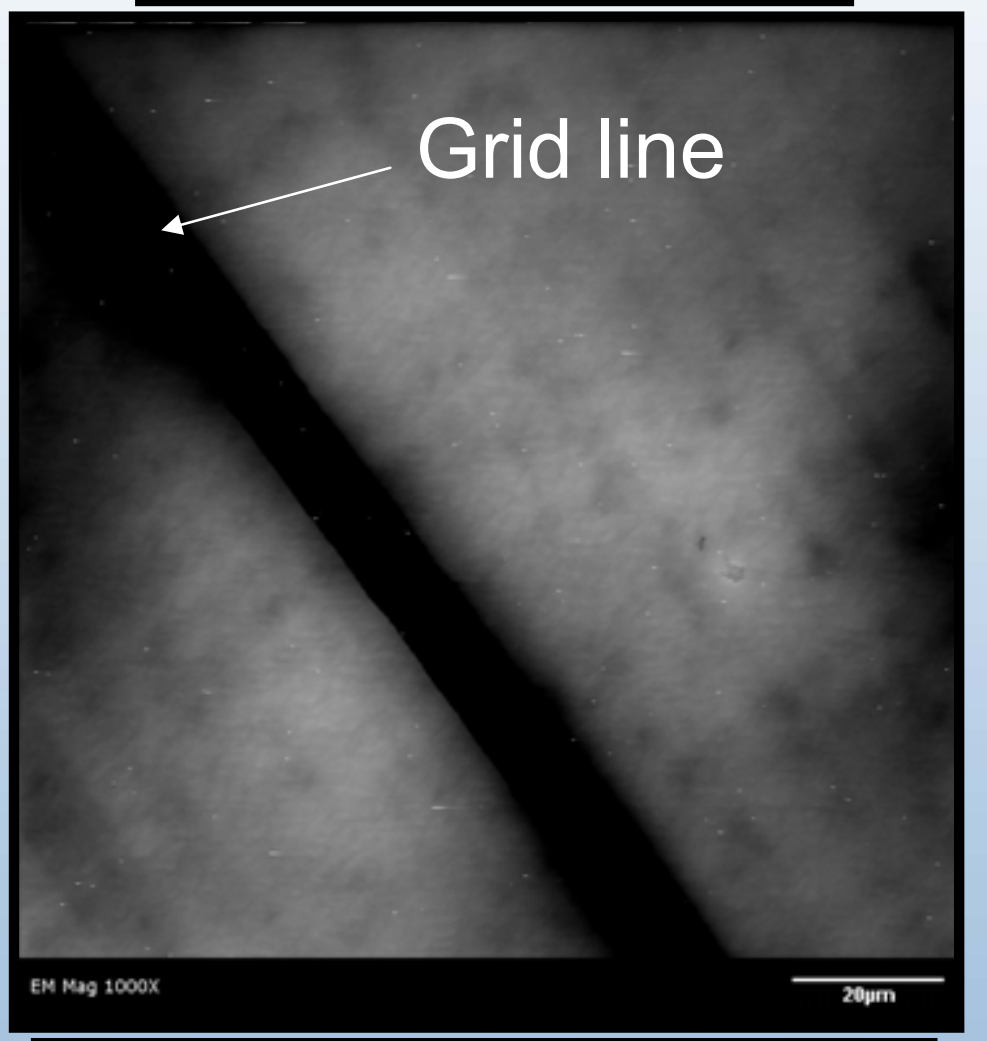

TD density $\sim 2 \mathrm{E} 6 \mathrm{~cm}^{-2}$ 


\section{1-eV, LMM (2.2\%) GalnAs/GalnP DH Cell Performance}

\section{Internal Quantum Efficiency}

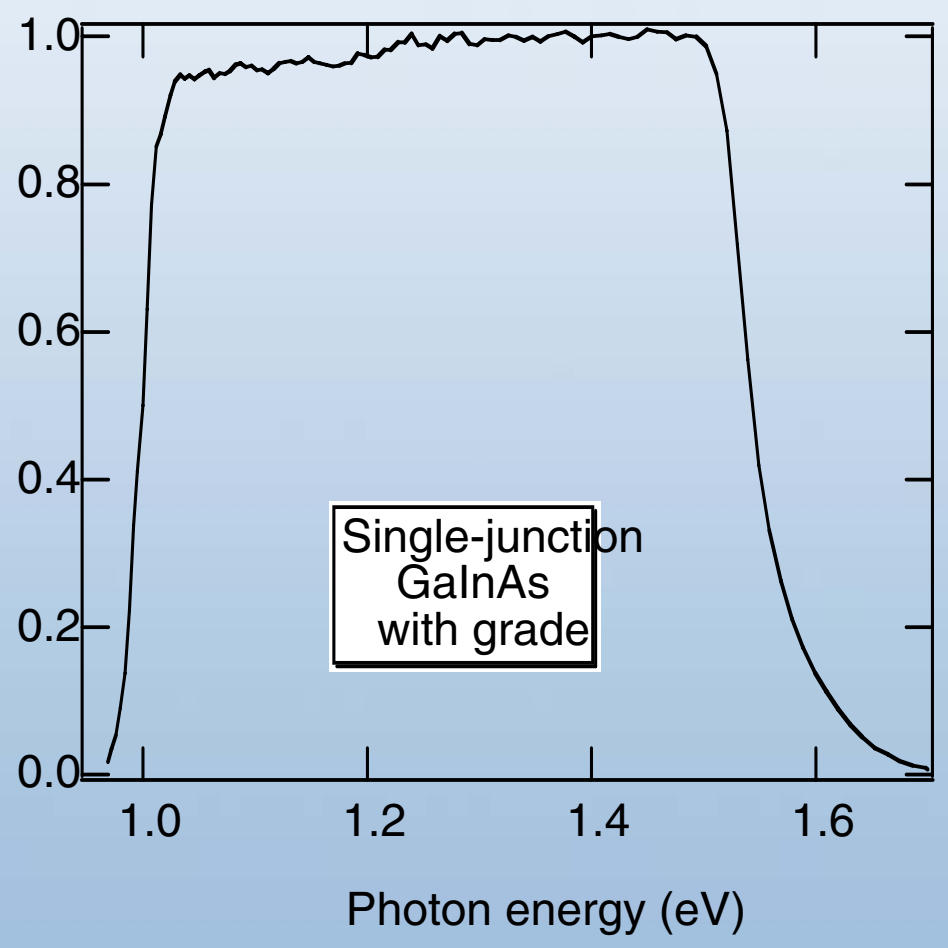

Current (Voltage)

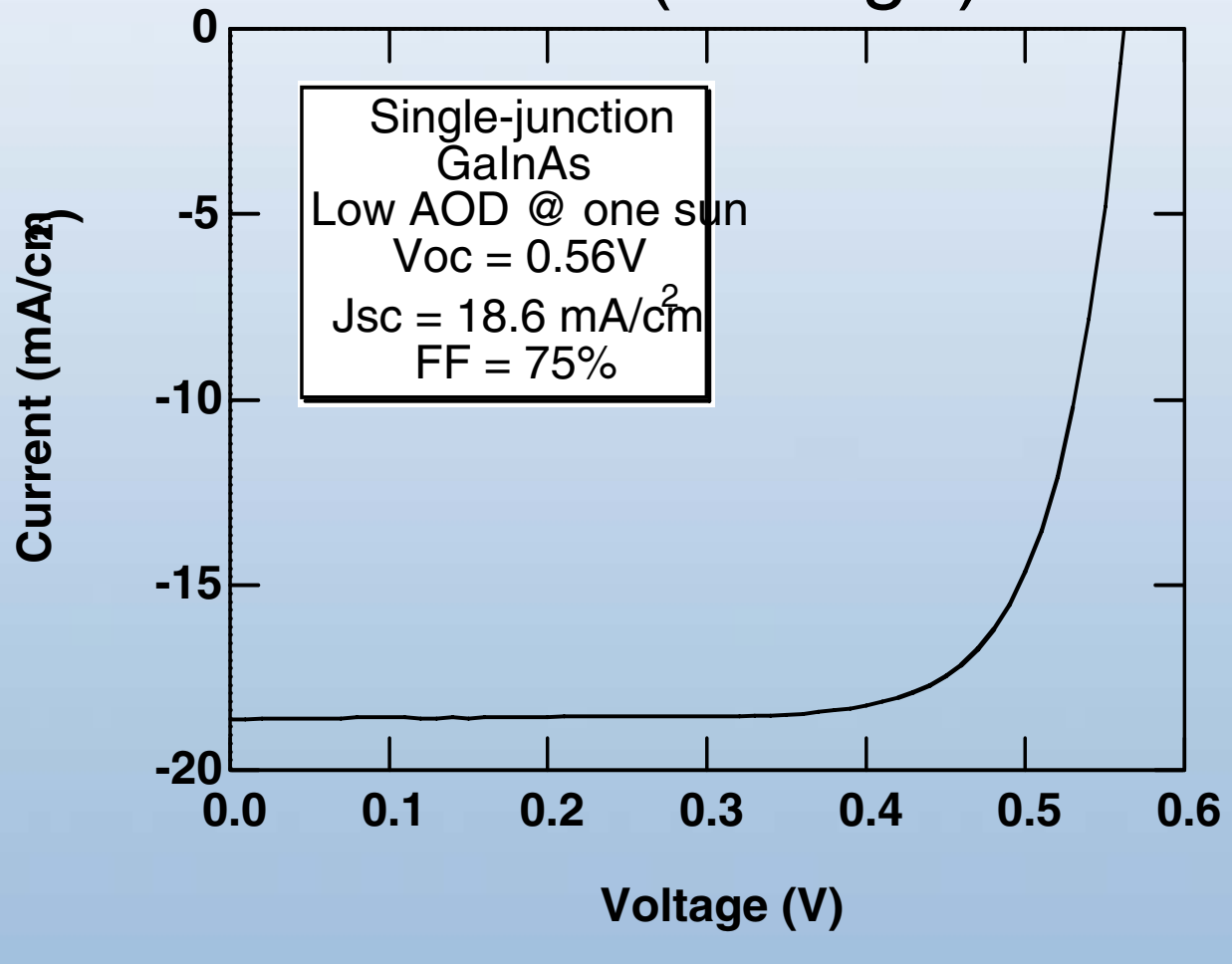




\section{Innovation for Our Energy Future}

\section{Ultra-Thin, Handle-Mounted \\ GalnP/GaAs/GalnAsTandem \\ AEQE \& R Data}

- QE is excellent for all subcells, but some improvement is still possible (reduce parasitic absorption and reflection).

- $\mathrm{ZnS} / \mathrm{MgF}_{2} \mathrm{ARC}$ is not optimal.

- Interference evident in thin bottom subcell.

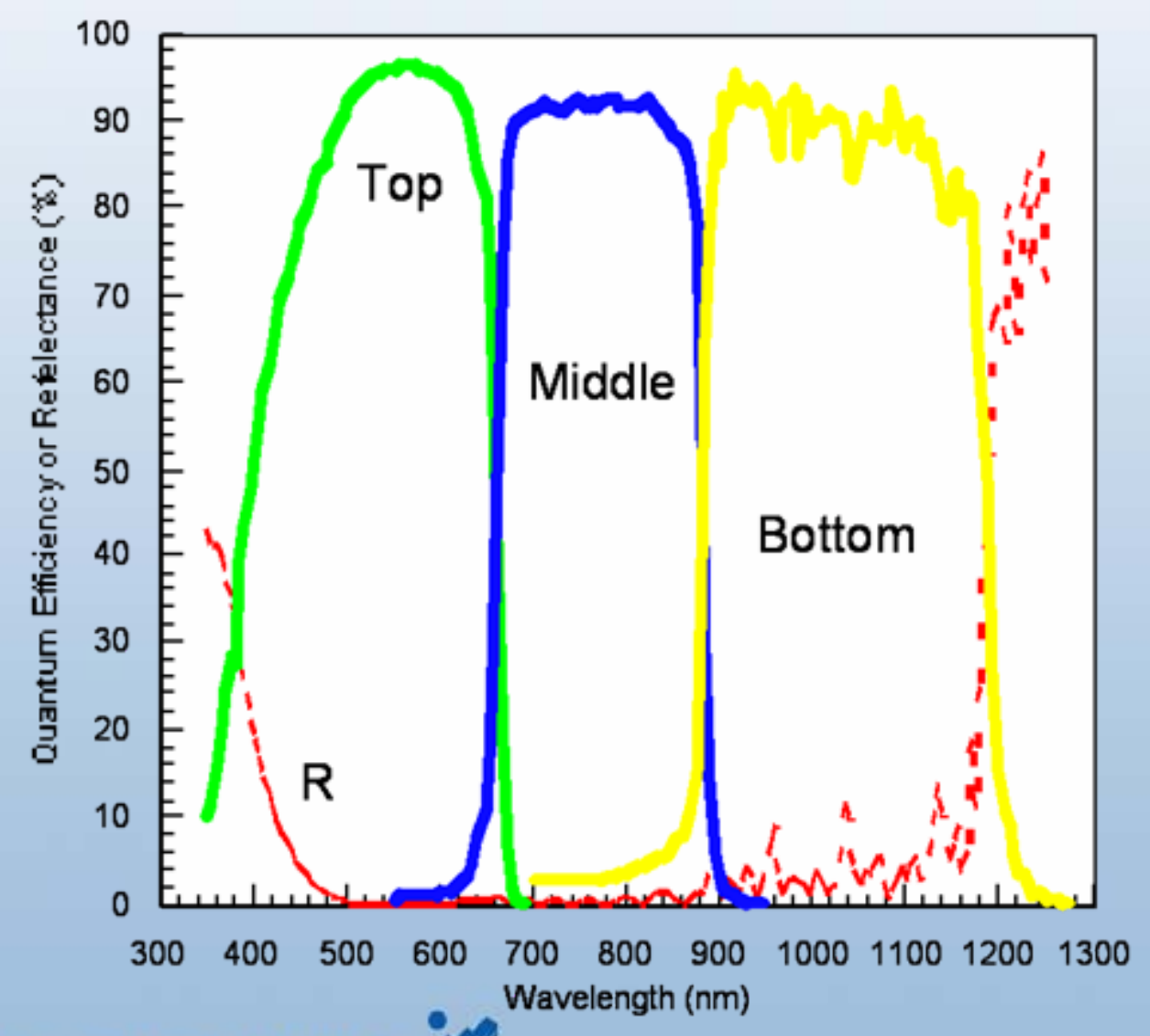




\section{NPEI National Renewable Energy Laboratory}

\section{Innovation for Our Energy Future}

\section{Reported Performance}

\begin{tabular}{|l|l|l|l|}
\hline PVSC & $1 / 3-7 / 05$ & Global, $25^{\circ} \mathrm{C}$ & $31.1 \%$ \\
\hline SPW & $4 / 18-21 / 05$ & AM0, 1 sun, $25^{\circ} \mathrm{C}$ & $29.7 \%$ \\
\hline ISCC & $5 / 1-5 / 05$ & Direct, 10.1 suns, $25^{\circ} \mathrm{C}$ & $37.9 \%$ \\
\hline SPRAT & $9 / 20-22 / 05$ & $\begin{array}{l}\text { AM0, } 1 \text { sun, } 25^{\circ} \mathrm{C} \\
\text { Kapton handle }\end{array}$ & $26.5 \%$ \\
\hline SPRAT & $9 / 20-22 / 05$ & AM0, 8.9 suns, $25^{\circ} \mathrm{C}$ & $31.4 \%$ \\
\hline
\end{tabular}




\section{$\mathrm{V}_{\mathrm{oc}}, \mathrm{FF}$ (Concentration Ratio)}

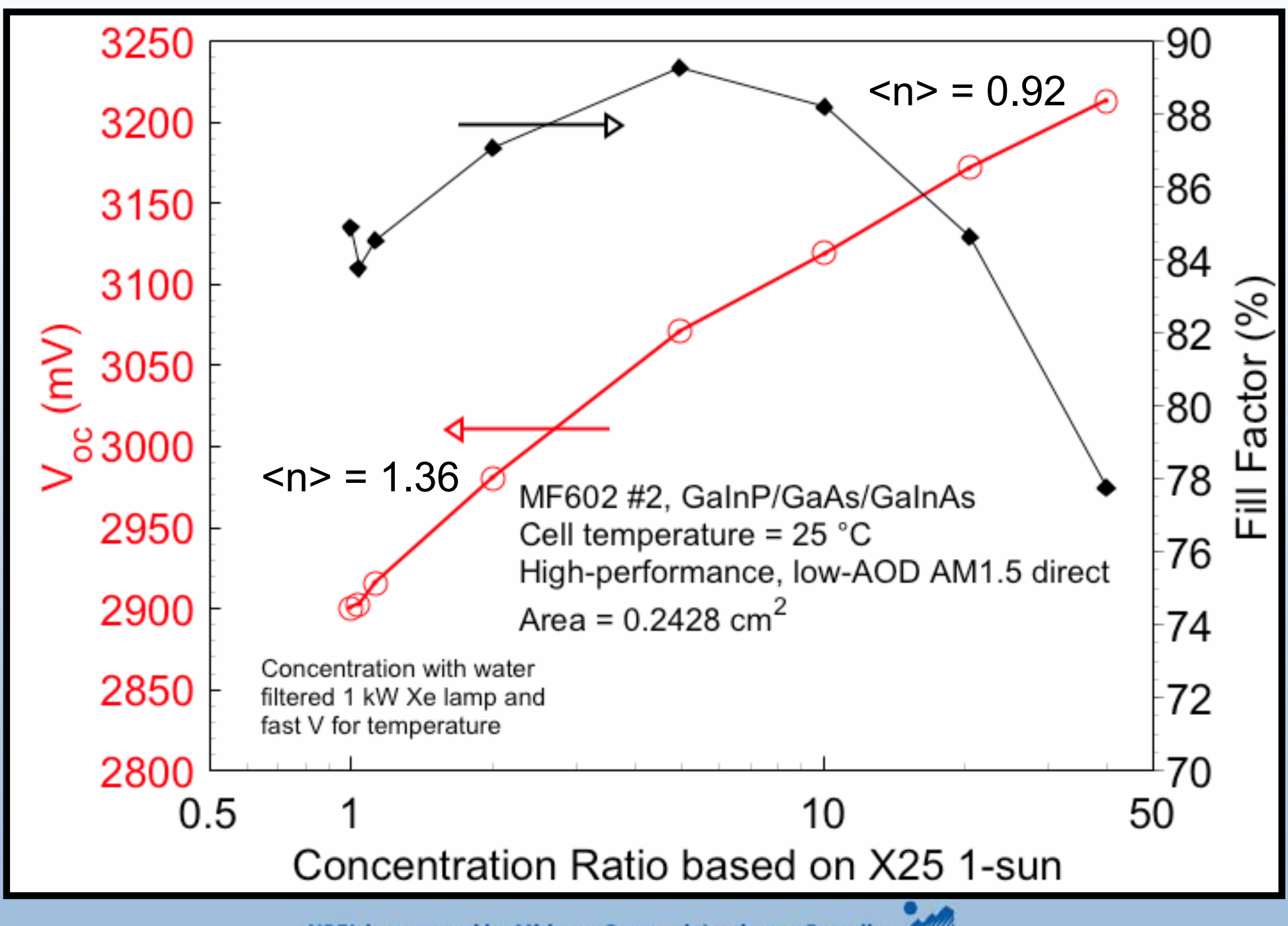




\section{I-V Data at \\ Peak Efficiency}

- $\eta=37.9 \%$ (10.1 suns).

- On 4/25/05, a new record for solar PV conversion.

- With continued development, $\eta>40 \%$ possible at higher concentration ratios.

\section{NREL}

GaInP/GaAs/GaInAs Cell

\section{Device ID: MF602 \#2}

Apr 25, 2005 2:26 PM

Device Temperature: $25.0 \pm 1.0^{\circ} \mathrm{C}$ Device Area: $0.2428 \mathrm{~cm}^{2}$

Reporting Spectrum: AM1.5 Direct

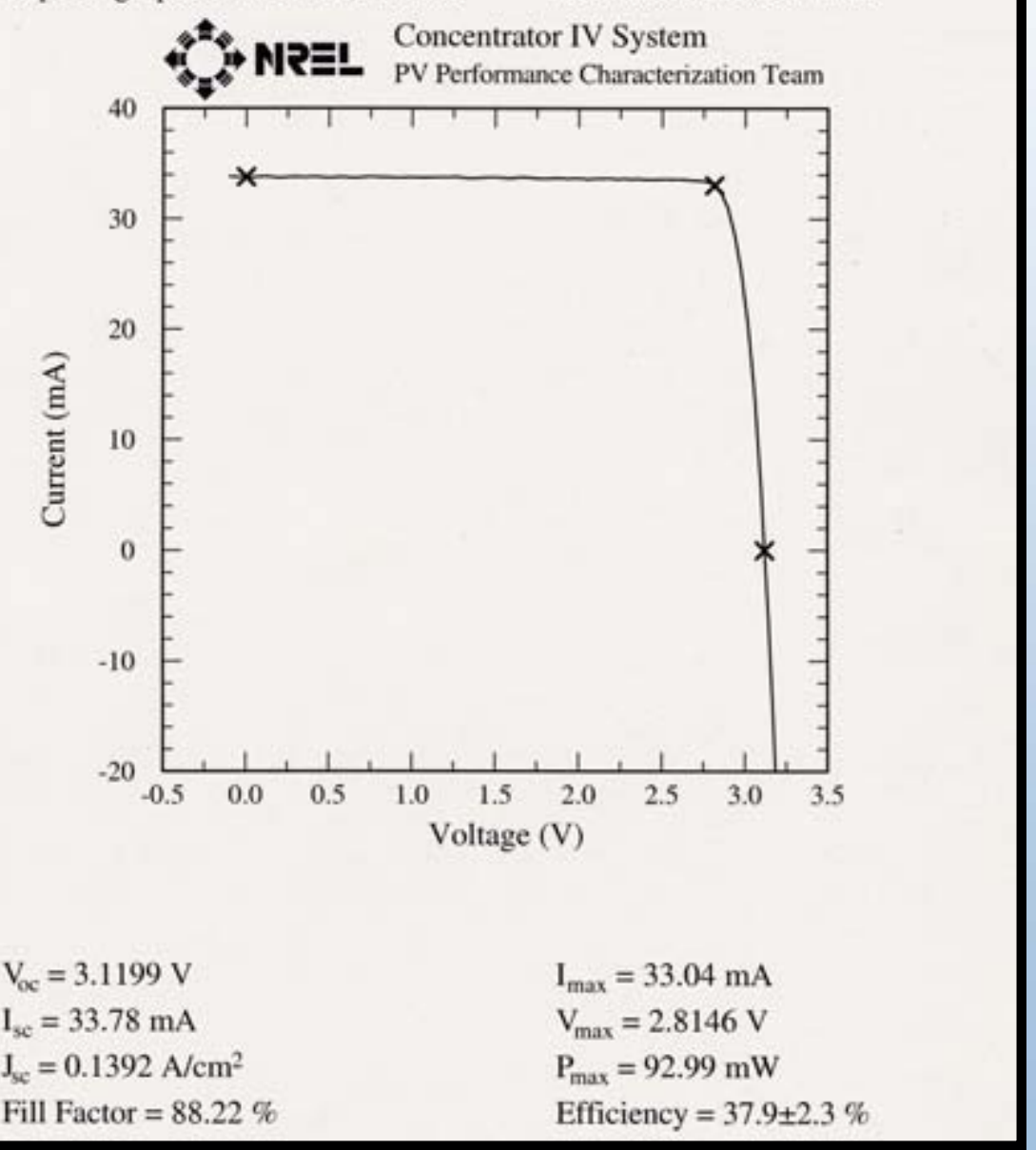




\section{Recent progress}

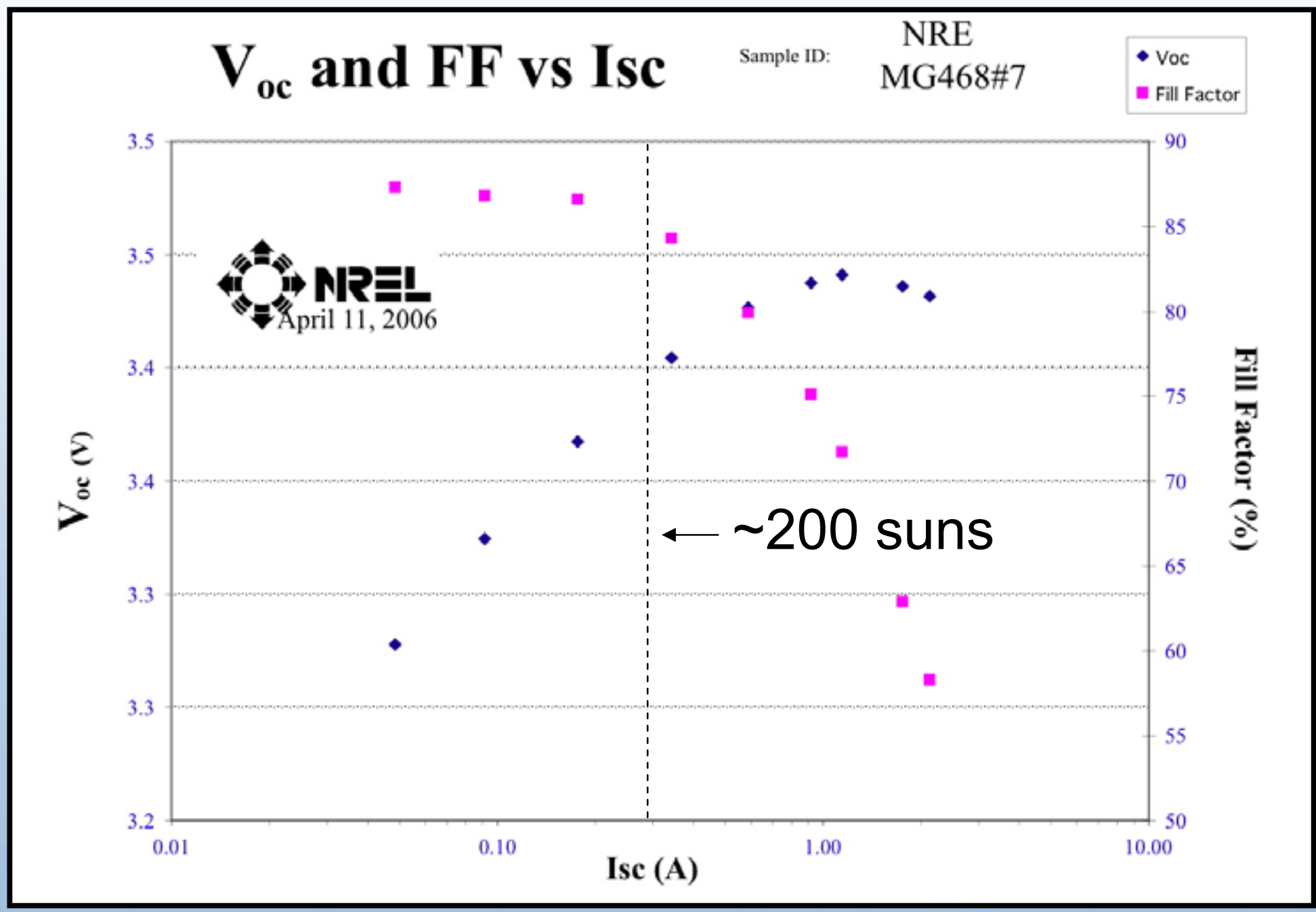




\section{Innovation for Our Energy Future}

\section{Research Issues}

- High-yield processing of handle-mounted, ultra-thin devices.

- Develop process enabling reuse of parent substrate.

- Tandem cell efficiency testing more difficult w/1-eV subcell even more difficult as we consider more than three subcells.

- Inverted tunnel junctions.

- Radiation effects.

- Push efficiency limits by including more subcells - can we achieve $40-50 \%$ (terr. conc.), 35-40\% (AM0)? 


\section{Advanced Design Options}

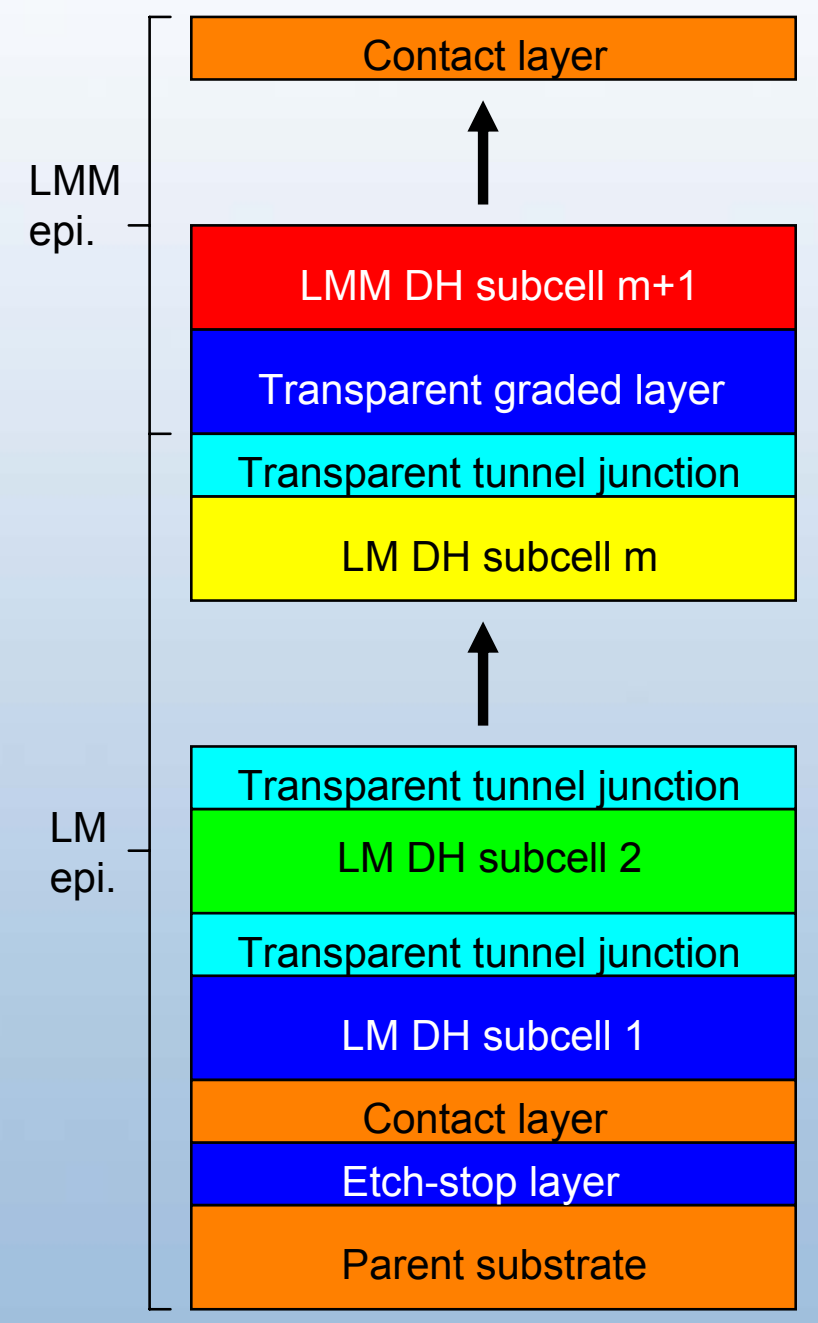

- Concept applies to two, or more, subcells.

- A wide range of substrates, subcell materials, tunnel junction materials, and transparent compositional grades are possible.

- Substrates: GaAs, Ge, Si, SiGe.

- Subcells, etc.: AlGalnPAsSb. 